\title{
A Review of the Genetics and Pathogenesis of Syndactyly in Humans and Experimental Animals: A 3-Step Pathway of Pathogenesis
}

\author{
Mohammad M. Al-Qattan (iD) \\ ${ }^{1}$ Professor of Hand Surgery, King Saud University, Riyadh, Saudi Arabia \\ ${ }^{2}$ King Faisal Specialist Hospital and Research Center, Riyadh, Saudi Arabia \\ Correspondence should be addressed to Mohammad M. Al-Qattan; moqattan@hotmail.com \\ Received 7 March 2019; Revised 23 August 2019; Accepted 1 September 2019; Published 15 September 2019 \\ Academic Editor: Przemko Tylzanowski
}

Copyright (c) 2019 Mohammad M. Al-Qattan. This is an open access article distributed under the Creative Commons Attribution License, which permits unrestricted use, distribution, and reproduction in any medium, provided the original work is properly cited.

Embryology of normal web space creation and the genetics of syndactyly in humans and experimental animals are well described in the literature. In this review, the author offers a 3-step pathway of pathogenesis for syndactyly. The first step is initiated either by the overactivation of the WNT canonical pathway or the suppression of the Bone Morphogenetic Protein (BMP) canonical pathway. This leads to an overexpression of Fibroblast Growth Factor 8 (FGF8). The final step is the suppression of retinoic acid in the interdigital mesenchyme leading to suppression of both apoptosis and extracellular matrix (ECM) degradation, resulting in syndactyly.

\section{Introduction}

Studies on developmental biology and embryology of the upper limb have greatly improved our understanding of the genetics and phenotypes of various congenital hand abnormalities [1].

The embryology of normal web space creation [2] and the genetics of human syndactyly [3] are well described in the literature. In this review, the author offers a 3-step pathway of pathogenesis for syndactyly. Based on the review, a classification of syndactyly will be offered based on these steps of pathogenesis.

\section{Classification of Syndactyly}

The genetics literature classifies syndactyly into nine types [3]. However, several other syndromic and nonsyndromic types of syndactyly are not included in this 9-type classification system. Examples include the Saudi-type familial syndactyly which is syntenic to the hammertoe locus in mice [4], the Cenani-Lenz syndactyly phenotype caused by APC mutations $[5,6]$, the brain atrophy-syndactyly syndrome caused by missense mutations of FIBULIN1 (OMIM 608180) [7], the triphalangeal thumb polysyndactyly syndrome caused by genomic duplications of the Sonic Hedgehog (SHH) enhancer ZRS (OMIM 174500) [8], acrocephalosyndactyly syndromes, Greig syndrome (OMIM 175700), and other syndactyly phenotypes associated with GLI3 mutations [9].

\section{The Unified Pathway of Pathogenesis of Syndactyly}

A 3-step pathway could explain the pathogenesis of almost all types of syndactyly in humans and experimental animals (Figure 1). The first step is either the activation of the WNT canonical signaling or the suppression of the Bone Morphogenetic Protein (BMP) canonical signaling. This will lead to an overexpression of Fibroblast Growth Factor 8 (FGF8) in the apical ectodermal ridge (AER) and also in the mesoderm since FGF8 is a diffusible morphogen [10]. This will then lead to the third step which is the suppression of 


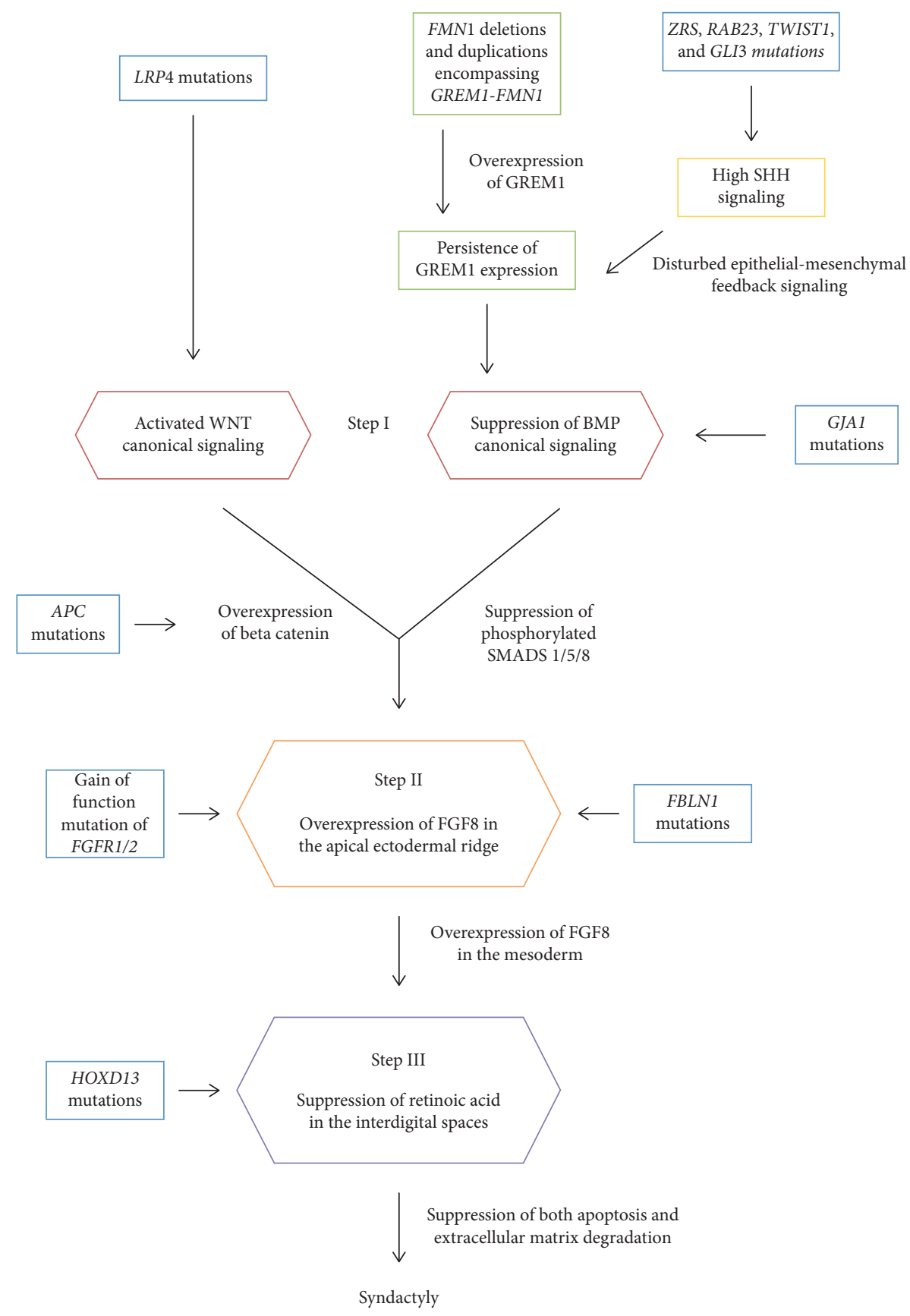

FIGURE 1: Human syndactyly and the 3-step pathway of pathogenesis.

retinoic acid in the interdigital spaces [11]. FGF8-retinoic acid interactions are mediated through the ERK/MAPK pathway $[12,13]$. The suppression of retinoic acid within the interdigital spaces will finally lead to syndactyly because of the suppression of both apoptosis and extracellular matrix (ECM) degradation. Normally, apoptosis is initiated by the action of proapoptotic proteins such as BAX and BAK on the mitochondria [14]. The promotor region of BAX and BAK contains retinoic acid responsive elements [15]. The normal disintegration of the ECM of the web space is mainly mediated by the ADAMTS family of peptidases and MMP11 $[16,17]$. The promotor region of the MMP11 gene also contains retinoic acid responsive elements [18]. Table 1 demonstrates that human syndactyly may be classified according to the 3 steps shown in Figure 1.

3.1. Step I: Related Syndactyly in Humans. LRP4 is a strong suppressor of WNT signaling, and hence, loss-of-function mutations of LRP4 will lead to signal activation and syndactyly (Cenani-Lenz syndrome, OMIM 212780) [19]. Lossof-function mutations of the APC gene lead to beta-catenin accumulation. In most cases, a familial adenomatous polyposis phenotype (OMIM 175100) is seen. However, APC mutations may occasionally result in a Cenani-Lenz syndactyly phenotype $[5,6]$. Similar Cenani-Lenz phenotypes 
TABle 1: Syndactyly in humans classified according to the steps shown in Figure 1.

\begin{tabular}{|c|c|c|}
\hline Step affected & Gene mutation & $\begin{array}{l}\text { Name of syndrome or type of syndactyly as } \\
\text { per Malik's classification (OMIM if available) }\end{array}$ \\
\hline \multirow{2}{*}{$\begin{array}{l}\text { IA: activation of the WNT canonical signaling } \\
\text { or the accumulation of beta catenin }\end{array}$} & (i) $L R P 4$ & $\begin{array}{l}\text { (i) Cenani-Lenz syndrome or type } 7 \mathrm{a} \\
\text { syndactyly }(212780)\end{array}$ \\
\hline & (ii) $A P C$ & (ii) Cenani-Lenz phenotype \\
\hline \multirow{5}{*}{ IB: suppression of the BMP canonical signaling } & $\begin{array}{l}\text { (i) FMN1 deletions or duplication } \\
\text { encompassing GREM1-FMN1 } \\
\text { (ii) ZRS }\end{array}$ & $\begin{array}{l}\text { (i) Cenani-Lenz phenotype or type } 7 \mathrm{~b} \\
\text { syndactyly } \\
\text { (ii) Haas (type 4) syndactyly (186200); } \\
\text { triphalangeal thumb polysyndactyly } \\
\text { syndrome (174500) }\end{array}$ \\
\hline & (iii) $R A B 23$ & (iii) Carpenter syndrome (201000) \\
\hline & (iv) TWIST1 & (iv) Saethre-Chotzen syndrome (101400) \\
\hline & (v) GLI3 & $\begin{array}{l}\text { (v) Greig syndrome (175700) and other } \\
\text { GLI3-related syndactyly }\end{array}$ \\
\hline & (vi) GJA1 & $\begin{array}{l}\text { (vi) Johnston-Kirby type } 3 \text { syndactyly } \\
\text { (186100) }\end{array}$ \\
\hline II: increased activity of FGF8 & $\begin{array}{l}\text { (i) Gain-of-function mutations of } \\
F G F R 1 \text { or FGFR2 } \\
\text { (ii) FBLN1 }\end{array}$ & $\begin{array}{l}\text { (i) Pfeiffer (101600), Apert (101200), and } \\
\text { Saethre-Chotzen (101400) syndromes. } \\
\text { (ii) Debeer type 2b syndactyly (608180) }\end{array}$ \\
\hline $\begin{array}{l}\text { III: suppression of retinoic acid or suppression of } \\
\text { apoptosis/matrix degradation }\end{array}$ & (i) $\mathrm{HOXD} 13$ & $\begin{array}{l}\text { (i) Vordingborg type 2a syndactyly (186000) } \\
\text { and syndactyly type } 5(186300)\end{array}$ \\
\hline
\end{tabular}

may also be caused by the overexpression of GREM1 (which is a strong suppressor of the BMP signaling) secondary to FMN1 deletions or duplications encompassing the GREM1FMN1 genes [20].

The normal events of the epithelial-mesenchymal feedback loop [21] participate in the process of normal web separation which occurs in humans between the $19^{\text {th }}$ and the $22^{\text {nd }}$ embryonic stages (47-54 intrauterine days) [22]. Prior to stage 19 , the digits are normally webbed. During this time, the high $\mathrm{SHH}$ activity stimulates the expression of GREM1, maintaining a high level of FGF8 and a low level of retinoic acid, and the digits are maintained in the webbed state. The growth of hand paddle during stages 19-22 leads to an increase in the gap between the $\mathrm{SHH}$ signal and GREM1-expressing cells. Hence, $\mathrm{SHH}$ is no longer able to stimulate GREM1 [21]. The result is stimulation of BMP signaling, suppression of FGF8, overexpression of retinoic acid, and finally web separation. Therefore, an abnormally high SHH activity in the limb bud will lead to persistence of GREM1 activity and syndactyly. In ducks and bats, the normally webbed digits/wings are caused by a persistent GREM1 expression [23]. In humans, gene mutations that lead to an increase in the activity of SHH will also lead to persistent GREM1 expression and syndactyly (Table 1). Point mutations/duplications within the ZRS or the surrounding region of $7 q 36$ lead to increased SHH activity and syndactyly [8, 24, 25]. RAB23, TWIST1, and GLI3 normally act as negative regulators of $\mathrm{SHH}$ [26-28]. Hence, loss-of-function mutations of RAB23, TWIST1, and GLI3 will be associated with a high SHH activity and syndactyly (Table 1 and Figure 1).

The last protein acting on Step I is the CX43 protein. Mutations of GJA1 lead to loss of the functional activity of CX43, which in turn leads to reduced BMPs and syndactyly (Syndactyly type 3, OMIM 186100) [29].
3.2. Step II: Related Syndactyly in Humans. In Step II, acrocephalo-syndactyly syndromes caused by gain-offunction mutations of FGFR1/FGFR2 are associated with an increased activity of FGF8 and syndactyly. Overexpression of other FGFs such as FGF2 in the cranium also occurs, leading to craniosynostosis. The severity of craniofacial abnormalities versus hand syndactyly will also depend on the type of mutation. A classic example is Apert syndrome (OMIM 101200). Hand surgeons classify patients with Apert syndrome with FGFR2 mutations into two groups according to the severity of syndactyly/craniofacial defects [30, 31]. The first group of patients have "severely affected" heads but "mildly affected" hands and carry the mutation Ser252Trp in FGFR2. The resulting gain-of-function of the receptor in this mutation leads to an increased functional effect of FGF2 which is mainly expressed in the craniofacial skeleton. The second group have "mildly affected" heads but "severely affected" hands and carry the mutation Pro253Arg in FGFR2. The resulting gain-of-function of the receptor in this mutation results in loss of the ligand-binding specificity in which the abnormal receptor is able to bind to FGF10, which results in upregulation of ectodermal FGF8 through the FGF10-FGF8 loop [31].

The pathophysiology of the synpolydactyly type $2 b$ (FBLN1 mutations, OMIM 608180) also occurs at Step II. Normally, the FIBULIN1 protein binds to FGF8 with high affinity, modulating its activity and expression [32]. Hence, the pathogenesis of this type of syndactyly is through Step II.

3.3. Step III: Related Syndactyly in Humans. HOXD13 mutations have been linked to two types of human syndactyly (Table 1) including the rare type 2 a Vordinborg synpolydactyly (OMIM 186000) and type 5 syndactyly (OMIM 186300) [33]. Experimental models have shown that the 
mutated Hoxd13 has a direct suppressive effect on retinoic acid in the autopod [34]. Hence, the pathogenesis of HOXD13-related syndactyly is through Step III (Figure 1 and Table 1). Brison et al. [35] reviewed the literature on HOXD13 mutations and found that the associated phenotypes included various forms of brachydactyly, syndactyly, and synpolydactyly. The pathogenesis of the polydactyly component in the Vordinborg synpolydactyly (OMIM 186000 ) is probably related to HOXD13-GLI3R interactions. Chen et al. [36] have shown experimentally that Hoxd13 directly binds to Gli3r (the repressor form of Gli3). The mutated Hoxd13 directs Gli3r for a premature degradation. This will result in polydactyly, similar to the polydactyly phenotype caused by depletion of Gli3r. Brison et al. [35] have also brought the attention that the G11A missense mutation of the HOXD13 gene in humans with synpolydactyly confirms a novel functional domain in HOXD13 which regulates digit number through its interaction with GLI3R.

3.4. Step I: Related Animal Models of Syndactyly. Animal models of syndactyly may also be classified according to the 3-step pathway of pathogenesis (Table 2).

Mice with Lrp4 mutations develop polysyndactyly in their forelimbs and hindlimbs [37]. The Lrp4 protein is a strong suppressor of WNT signaling, and hence, loss-offunction mutations of $L r p 4$ will lead to signal activation and syndactyly [37].

The murine limb deformity (Ld) model is caused by Fmn1 deletions and show oligosyndactyly, renal defects, and radio-ulnar synostosis [38]. The phenotype of the Ld model is considered to be the closest phenotype to the phenotype of Cenani-Lenz syndrome in humans. The phenotype of transgenic chicks with Grem1 overexpression [39] is also similar to the human syndactyly phenotype associated with duplications encompassing the GREM1 gene [20].

Other animal models of syndactyly were created by suppression of Bmp signaling. Overexpression of the Bmp antagonist Noggin in mice resulted in extensive soft-tissue syndactyly and postaxial polydactyly [40]. Inactivation of the mouse Bmp receptor gene Bmprla in the limb bud was associated with upregulation of both Fgf8 and Fgf4, resulting in syndactyly [41]. Bmp2-deficient mice display soft-tissue syndactyly of the third web space, while combined deficiency of Bmp2 and Bmp4 in the limb bud results in complete syndactyly of all limbs [42]. Mice lacking Cx43 show a reduction in the expression of Bmp2 leading to a secondary overexpression of Fgfs and syndactyly [29]. SMADs 1 and 5 are downstream of the BMP signaling. Selective inactivation of Smads 1 and 5 in mice results in overexpression of Fgf8 and syndactyly [43].

Finally, the hammertoe $(\mathrm{Hm})$ mutant mouse model shows syndactyly of digits $2-5$. In this spontaneous mouse mutation, a $150 \mathrm{~kb}$ noncoding DNA fragment from chromosome 14 is inserted upstream of the Shh promotor. This results in overexpression of interdigital Shh, secondary suppression of the Bmp signaling, and finally syndactyly. The pathophysiology of this secondary suppression of Bmp was recently shown by Mouri et al. [44]. The overexpression of Shh leads to the upregulation of Chordin. Chordin binds to Bmps and sequestrates them into latent complexes, thereby suppressing the Bmp activity [44].

\subsection{Step II: Related Animal Models of Syndactyly.} Suppression of notch signaling in mice results in increased expression of Fgf8 in the AER and syndactyly [45]. Notch 1 and its ligand Jagged 2 are coexpressed in the AER. Suppression of Notch signaling has been demonstrated in mice lacking Jagged2. This resulted in an increased expression of Fgf8 and fusion of the middle three digits. Since Notch is also expressed in the thymus and the craniofacial area, mutant mice also exhibited cleft palate, tongue fusion, and thymic defects [46].

Msx1/Msx2 double-mutant mice show variable phenotypes including oligodactyly, polydactyly, and syndactyly. This is associated with extended Fgf activity in the AER [47].

The FRAS1 gene encodes an extracellular matrix protein involved in the establishment of the epidermal basement membrane. Mutations in FRAS1 in humans cause Fraser syndrome (OMIM 219000) with eye, kidney, and craniofacial defects [48]. Hines et al. [49] identified a novel ENUderived rounded foot (rdf) mouse mutant with hindlimb cutaneous syndactyly caused by loss-of-function nonsense allele of Fras1. The primary defect in these animals was the decreased Msx2 expression [49]. As mentioned above, deficiency of Msx1/2 is associated with extended Fgf activity in the AER [47].

Basement membranes are extracellular matrices underlying the epithelium and endothelium of various organs including the AER. All basement membranes contain at least one member of Laminin, type IV collagen, and Nidogen families [50]. Two Nidogen isoforms (Nidogens 1 and 2) have been identified in vertebrates. The individual knockout of either Nid1 or Nid2 in mice does not affect basement membrane formation and animals show no abnormalities. Mice lacking both Nidogens have defective ectodermal basement membrane of multiple organs including aberrant AER formation, altered distribution of Fgf8, and soft-tissue syndactyly [51]. Since basement membrane formation of multiple organs are affected, perinatal lethality with multiorgan defects are also seen in these animals [50]. This model shows that altered distribution of Fgf8 may also lead to syndactyly.

3.6. Step III: Related Animal Models of Syndactyly. Animal models with suppressed retinoic acid activity show syndactyly $[17,18]$. The retinaldehyde dehydrogenase- 2 enzyme is encoded by the Raldh2 gene. The enzyme oxidizes retinaldehyde to retinoic acid. Retinoic acid functions as a ligand for nuclear retinoic acid receptors (known as RAR) to induce the transcription of target genes. Hence, suppression of retinoic acid activity in experimental mice may be done either by targeting the retinaldehyde dehydrogenase- 2 enzyme or the RAR receptors. Raldh $2^{-1-}$ autopods show syndactyly secondary to suppression of Mmp11 (which is responsible for interdigital ECM degradation). In this 
TABLE 2: Syndactyly in animal models classified according to the steps shown in Figure 1.

\begin{tabular}{ll}
\hline Step affected & Animal model of syndactyly \\
$\begin{array}{l}\text { IA: activation of the WNT canonical signaling or the } \\
\text { accumulation of beta catenin }\end{array}$ & Mice with Lrp4 mutations \\
\hline & (i) Murine limb deformity (Ld) model (Fmn1 \\
deletions resulting in Grem1 overexpression) & (ii) Transgenic chick with Grem1 overexpression \\
IB: suppression of the BMP canonical signaling or the & (iii) Overexpression of the Bmp antagonist Noggin \\
suppression of BMPs/SMADs & (iv) Inactivation of the Bmp receptor gene Bmpr1a \\
& (v) Mice deficient in Bmp2 and Bmp4 \\
& (vi) Knockout mice lacking Cx43 \\
(vii) Selective inactivation of Smad 1 and 5. & (viii) Hammer toe (Hm) mutant mice.
\end{tabular}

(i) Suppression of Notch signaling

(ii) Msx1; Msx2 double-mutant mice

II: overexpression of FGF8

(iii) Rounded foot mouse mutants (Fras1 loss-offunction)

(iv) Mice lacking Nidogen 1 and 2.

(i) Suppression of retinoic acid activity

III: suppression of retinoic acid or suppression of

(ii) $\mathrm{Spdh} / \mathrm{Spdh}$ mice

apoptosis/extracellular matrix degradation

(iii) Bid, Bim, Puma triple knockout mice

(iv) Mice deficient in ADAMTS

(v) Knockout of Akirin2 in the limb ectoderm

model, Fgf8 expression is normal, indicating that retinoic acid acts downstream of Fgf8 in the pathogenesis of syndactyly [17]. $R A R b / R A R g$ double-mutant mice also show syndactyly secondary to the suppression of Mmp11 [18].

Kuss et al. [34] used the naturally occurring mouse mutant (Spdh/Spdh mutant) that has the polyalanine expansions in homeobox d13 (Hoxd13). The authors showed that the mutated Hoxd13 has a direct suppressive effect on retinoic acid in the autopod [34]. Intrauterine treatment with retinoic acid restored pentadactyly in Spdh/Spdh mice [34].

The proteins Bid, Bim, and Puma act to activate Bax and Bak which are essential to initiate the apoptotic pathway at the mitochondrial level. Hence, Bid/Bim/Puma triple knockout mice show reduced apoptosis and syndactyly. Besides Mmp11, the ADAMTS group of peptides is essential in interdigital ECM degradation. Versican (a proteoglycan) is an important component of the interdigital ECM. ADAMTS mediates the cleavage of versican. Hence, mice deficient in ADAMTS exhibit syndactyly [16].

Akirins (Akirin 1 and 2) are small nuclear proteins that localize to promoter and enhancer regions of genes. They function as "bridge" proteins to coordinate gene expression patterns [52]. Akirin2 null embryos are not able to survive beyond embryonic day 9.5. Transgenic mouse models with knockout of Akirin2 in the limb epithelium leads to a loss of interdigital cell death and an increase in cell proliferation, resulting in the retention of the interdigital web and softtissue syndactyly [52].

\section{Discussion}

Limb development is a complex process involving the action of signaling centers that coordinate spatially and temporally to sculpt a limb [22]. Digit formation requires the combined coordination of morphogen gradients and feedback loops that dictate responses by cells of the AER, zone of polarizing activity in which SHH is expressed, nonAER ectoderm, and mesenchymal cells within the limb bud. Finally, the regulation of interdigital tissue regression also requires the interplay of multiple spatiotemporally controlled morphogen gradients to ensure proper limb formation and release of individual digits. Understandably, syndactyly may originate when there is a failure in the regulation of interdigital tissue regression. The limb morphogenesis is better understood when the genetic networks are categorized with respect to well-established proximodistal, anteroposterior, and dorsoventral axes [22]. FGF8, SHH, and WNT7A/EN1 are the main controllers of these 3 axes, respectively. Congenital limb defects may be classified according to the axis defect. For example, brachydactyly and amelia are related to defects in the proximodistal axis; polydactyly as well as radial/ulnar ray deficiencies are related to defects in the anteroposterior axis; and ventral/dorsal dimelia (the appearance of palmar structures on the dorsal aspect of the hand and the appearance of dorsal structures on the ventral aspect of the hand, respectively) are related to defects in the dorsoventral axis $[53,54]$. Hence, it would be pertinent to understand the origin of syndactyly with respect to the developmental axis. Oberg [53] studied this extensively and concluded that syndactyly is best classified under malformations of "unspecified" axis [53]. However, since FGF8 is the key middle step mediating the pathogenesis of syndactyly, one may argue that syndactyly may be considered as a defect in the proximodistal axis (FGF8 is the main controller of this axis).

The current review offers a unified pathway that could explain the pathogenesis of syndactyly in humans and experimental animals. The pathway is a 3-step pathway, and hence, human and animal syndactyly may be classified accordingly (Tables 1 and 2). The final step in the pathway is 
the suppression of retinoic acid in the mesenchyme which will lead to the suppression of both apoptosis and ECM degradation. It is important to realize that retinoic acid only induces apoptosis/EMC degradation within the interdigital space and not within the digits. The explanation for this was shown by Zhao et al. [17]. The digits (and not the interdigital spaces) express a cytochrome known as Cyp26b1 which inactivates retinoic acid. Hence, mesodermal retinoic acid is unable to "degrade" the digits.

The pathway proposed in the current review explains the pathogenesis of most types/models of syndactyly. Certain types of syndactyly have a different pathway of pathogenesis (such as Poland syndrome syndactyly) or the pathogenesis is yet to be determined (such as Shaker syndactyly mouse models, syndactyly related to b-HLHA9 in both humans and animals, syndactyly of Noggin-null mice, syndactyly of Sp6 mutant mice, and isolated 4/5 metacarpal fusion in humans). These types of syndactyly are summarized in Table 3.

Poland syndrome (OMIM 173800) is characterized by the unilateral absence or hypoplasia of the sternocostal head of the pectoralis major muscle and ipsilateral symbrachydactyly. However, several other pectoral muscle and hand defects have also been described [55]. One characteristic radiological feature in the hands of these patients is the pronounced hypoplasia or aplasia of the middle phalanx [56]. Poland syndrome results from a vascular insult to the subclavian artery during the 19th embryonic stage. During this stage, three events normally occur: the development of the sternocostal head of the pectoralis major, the chondrification of the middle phalanges and the initial phase of finger separation [57]. Hence, the pathogenesis of syndactyly in Poland syndrome is not related to our 3-step pathway.

The mouse mutant Shaker-with-syndactylism (Sy) is caused by Fibrillin2 (Fbn2) loss-of-function mutations [58]. Fibrillin2 is an important component of elastic fibers and the pathophysiology of "Sy" syndactyly is unknown. However, Fibrillins are known to interact with Fiblulins [59]. Since FIBULIN1 deficiency leads to increased FGF8 activity and syndactyly (see Table 1), these interactions may explain the pathogenesis of syndactyly in Shaker mice.

The Basic Helix-Loop-Helix Member A9 (b-HLHA9, also known as FINGERIN) is a transcription factor which is expressed in the distal hand/foot plates [60]. Duplications of BHLHA9 result in split hand-foot malformation with longbone deficiency type 3 (SHFLD 3, OMIM 612576) [61]. In contrast, loss-of-function mutations of BHLHA9 lead to mesoaxial synostotic syndactyly with phalangeal reduction (MSSD, Type 9 human syndactyly, OMIM 609432) [3]. Schatz et al. [60] have established that complete deletion of the murine ortholog of Fingerin causes syndactyly, both in vivo and ex vivo. However, the exact pathogenesis of syndactyly is still unknown, and two different theories have been proposed [60]. The first theory is based on the fact that b-HLHA9 is a down-stream target of Notch signaling [62]. As mentioned above (see Table 2), suppression of Notch signaling leads to an increased expression of Fgf8 in the AER and syndactyly [45]. The second theory is based on the fact that b-HLHA9 contains a unique proline-rich carboxyterminus not typically found in other bHLH factors [60].
Proline-rich domains participate in protein-protein interactions which may sequester the function of proteins that provide the apoptotic signal [60].

Mutations in NOGGIN in humans leads to various phenotypes including brachydactyly type B2 (OMIM 611377) and various forms of synostosis/ankylosis/symphalangism (OMIM 186500, 184460, 185800, 186570). In contrast, cutaneous syndactyly is a prominent feature in Noggin-null mice [63]. The exact pathogenesis of syndactyly is unknown but is thought to be related to the secondary overexpression of Indian hedgehog (Ihh) in the interdigital spaces, which in turn leads to reduced apoptosis [63].

Sp6 (also known as Epiporfin) and Sp8 (also known as Buttonhead) are transcription factors implicated in AER induction and maintenance [64]. They are expressed in the limb bud ectoderm and AER and induce the expression of Fgf8 in the AER [64]. Sp8 mutant mice exhibit severe loss of Fgf8 expression in the AER leading to limb truncations. In contrast, Sp6 mutant mice show a mild loss of Fgf8 expression and a mild syndactyly phenotype [64]. The pathogenesis may be explained by the fact that animal models with mild deficiency of Fgf8 in the AER show secondary overexpression of Fgf4 (normally, Fgf4 is restricted to the posterior AER) [65]. As a result of this ectopic Fgf4 expression, all of the skeletal defects caused by the loss of Fgf8 are rescued, and syndactyly is seen in the phenotype of experimental animals [66]. This pathogenesis also explains the occasional presence of syndactyly instead of ectrodactyly in one of the limbs in patients with split hand-foot malformations (which are associated with suppression of FGF8 in the AER) $[67,68]$.

Isolated fusion of the $4 / 5$ metacarpal has been classified as syndactyly type 8 (OMIM 309630). This is the only type of syndactyly that is inherited as X-linked and is caused by nonsense mutations in FGF16 [69]. The pathogenesis of the isolated metacarpal fusion is unknown. However, Fgf16 knockdown in experimental animals is known to affect Fgf8 expression in the AER as well as the expression of Shh expression in the zone of polarizing activity [70].

Our review also demonstrates that mutations in humans and animals do not always result in similar phenotypes. Fbn2 mutations in mice show syndactyly, while FBN2 mutations in humans are associated with congenital contractual arachnodactyly (OMIM 121050). Another example is the mouse model of Apert syndrome which showed most of the classic craniofacial features, but none of the animals exhibited syndactyly [71]. A third example is the phenotype associated with the loss of SMADs (SMADs 1/5/8-SMAD 4 complexes act as transcription factors for the BMP signaling pathway). In animals, deficiency of Smads $1 / 5$ results in a syndactyly phenotype [43]. In humans, no specific syndromes are related to SMAD 1/5 deficiency. Instead, loss-offunction mutations of SMAD 4 in humans are associated with Juvenile polyposis syndromes (OMIM: 174900 \&175050).

Another observation from the current review is the correlation between the degree of pathway abnormality and the severity of syndactyly phenotype. One example is seen in Apert patients. The "severely affected" hand group has a 
TABLE 3: Other types of syndactyly with a unique pathway of pathogenesis or with an undetermined pathogenesis.

\begin{tabular}{|c|c|c|c|}
\hline Type of syndactyly & $\begin{array}{l}\text { Animals or } \\
\text { humans }\end{array}$ & Gene mutation/knockout & $\begin{array}{c}\text { Proposed pathogenesis as per our } \\
\text { literature review }\end{array}$ \\
\hline Poland syndrome (OMIM 173800) & Humans & - & $\begin{array}{l}\text { Unique pathway: vascular insult to the } \\
\text { subclavian artery during the } 19^{\text {th }} \\
\text { embryonic stage }\end{array}$ \\
\hline Shaker syndactyly & Mice & Fbn2 & $\begin{array}{l}\text { Fibrillin-Fibulin interactions leading to } \\
\text { increased Fgf8 activity (step II) }\end{array}$ \\
\hline $\begin{array}{l}\text { Type } 9 \text { human syndactyly (OMIM } \\
\text { 609432) and mice deficient in BhLha9 }\end{array}$ & Humans/mice & $\begin{array}{l}\text { Loss-of-function mutations of } \\
\text { BHLHA9 and deletion of the murine } \\
\text { ortholog Bhlha9 }\end{array}$ & $\begin{array}{c}\text { Either through suppression of Notch } \\
\text { signaling (step II, see Table 2) or } \\
\text { disturbance of the apoptotic signal } \\
\text { (step III) }\end{array}$ \\
\hline Noggin-null mice & Mice & Nog & $\begin{array}{l}\text { The overexpression of Ihh in the } \\
\text { interdigital spaces leading to reduced } \\
\text { apoptosis (step III) }\end{array}$ \\
\hline Sp6 mutant mice & Mice & Sp6 & $\begin{array}{l}\text { The mild deficiency in Fgf8 leads to } \\
\text { overexpression of Fgf } 4 \text { in the AER } \\
\text { resulting in syndactyly }\end{array}$ \\
\hline $\begin{array}{l}\text { Type } 8 \text { human syndactyly (OMIM } \\
309630 \text { ) }\end{array}$ & Humans & FGF16 & $\begin{array}{c}\text { Either via altering the expression of } \\
\text { FGF8 or SHH }\end{array}$ \\
\hline
\end{tabular}

AER: apical ectodermal ridge.

more severe syndactyly phenotype and higher FGF8 overactivity when compared to the "mildly affected" hand group. Another example is Cenani-Lenz syndrome (OMIM 212780) caused by LRP4 mutations. The classic syndrome is characterized by bilateral hand and feet syndactyly along with metacarpal/metatarsal synostosis and phalangeal disorganization. Hand function in these patients is poor even after surgical correction [72]. This relatively severe classic syndactyly phenotype is associated with homozygous and compound heterozygous missense and splice mutations of the LRP4 gene [19]. In contrast, missense mutations of the LRP4 gene cause a very mild phenotype with simple syndactyly and well-developed digits, without metacarpal synostosis/phalangeal disorganization [73]. Furthermore, complete loss-of-function mutations of LRP4 lead to the most severe oligosyndactyly phenotype and may even be lethal with intrauterine demise [74-76]. A third example is seen in Type 2a human synpolydactyly (OMIM 186000). A mild syndactyly phenotype is seen with heterozygous HOXD13 mutations, and a severe syndactyly phenotype is seen with homozygous HOXD13 mutations [33, 77].

Finally, the author noted that the syndactyly phenotype related to LRP4 and APC mutations (which directly act on the WNT canonical pathway in Step I) and those directly leading to persistence of GREM1 expression (which directly act on the BMP canonical pathway in Step I) will have a similar Cenani-Lenz phenotype. Hence, syndactyly related to mutations of LRP4, APC, FMN1, and GREM1 should be grouped together. In contrast, human syndactyly related to high $\mathrm{SHH}$ signaling has a different syndactyly phenotype and is associated with preaxial polydactyly, although the pathogenesis is still at Step I (Figure 1). This may be explained by the fact that the high $\mathrm{SHH}$ is acting indirectly on GREM1 (via the epithelial-mesenchymal feedback loop). Furthermore, the high $\mathrm{SHH}$ expression is expected to result in ectopic anterior expression of $\mathrm{SHH}$, leading to concurrent preaxial polydactyly [78]. This explains the phenotype of syndactyly and concurrent polydactyly in Haas (OMIM 186200), triphalangeal thumb polysyndactyly (OMIM 174500), Carpenter (201000), Saethre-Chotzen (OMIM 101400), and Greig (OMIM 175700) syndromes (Table 1).

\section{Conclusions and Future Perspectives}

The 3-step pathway of pathogenesis provides a novel look at the correlation between the genetics and pathogenesis of syndactyly. The syndactyly is best classified according to the pathogenesis since the phenotype is affected by the site along the pathway. The pathway may also help to guide research on syndactyly types with undetermined pathogenesis. For example, the mouse mutant Shaker-with-syndactylism (Sy) is caused by Fibrillin2 (Fbn2) loss-of-function mutations [58]. Since Fibrillins and Fibulins are both important components of elastic fibers and both are known to interact [59], the pathogenesis of Sy syndactyly may be investigated to determine if it is through Fibulin1 as a modifier of Fgf8 activity in Step II.

\section{Conflicts of Interest}

The author declares no conflicts of interest.

\section{Acknowledgments}

This work was supported by the College of Medicine Research Center, Deanship of Scientific Research, King Saud University, Saudi Arabia.

\section{References}

[1] M. K. Richardson, "Molecular tools, classic questions-an interview with Clifford Tabin," The International Journal of Developmental Biology, vol. 53, no. 5-6, pp. 725-731, 2009.

[2] M. M. Al-Qattan, "Formation of normal interdigital web spaces in the hand revisited: implications for the pathogenesis 
of syndactyly in humans and experimental animals," Journal of Hand Surgery (European Volume), vol. 39, no. 5, pp. 491498, 2014.

[3] S. Malik, "Syndactyly: phenotypes, genetics and current classification," European Journal of Human Genetics, vol. 20, no. 8, pp. 817-824, 2012.

[4] M. M. Al-Qattan, H. E. Shamseldin, M. Al Mazyad, S. Al Deghaither, and F. S. Alkuraya, "Genetic heterogeneity in type III familial cutaneous syndactyly and linkage to chromosome 7q36," American Journal of Medical Genetics Part A, vol. 161, no. 7, pp. 1579-1584, 2013.

[5] N. Patel, E. Faqeih, S. Anazi et al., "A novel APC mutation defines a second locus for Cenani-Lenz syndrome," Journal of Medical Genetics, vol. 52, no. 5, pp. 317-321, 2015.

[6] M. M. Al-Qattan and F. S. Alkuraya, "Cenani-Lenz syndrome and other related syndactyly disorders due to variants in LRP4, GREM1/FMN1, and APC: insight into the pathogenesis and the relationship to polyposis through the WNT and BMP antagonistic pathways," American Journal of Medical Genetics Part A, vol. 179, no. 2, pp. 266-279, 2018.

[7] S. Bohlega, H. Al-Ajlan, and A. Al-Saif, "Mutation of fibulin-1 causes a novel syndrome involving the central nervous system and connective tissues," European Journal of Human Genetics, vol. 22, no. 5, pp. 640-643, 2014.

[8] M. Sun, F. Ma, X. Zeng et al., "Triphalangeal thumb-polysyndactyly syndrome and syndactyly type IV are caused by genomic duplications involving the long range, limb-specific SHH enhancer," Journal of Medical Genetics, vol. 45, no. 9, pp. 589-595, 2008.

[9] M. M. Al-Qattan, "A novel frameshift mutation of theGLI3gene in a family with broad thumbs with/without big toes, postaxial polydactyly and variable syndactyly of the hands/feet," Clinical Genetics, vol. 82, no. 5, pp. 502-504, 2012.

[10] M. Villacorte, K. Suzuki, K. Hayashi et al., "Antagonistic crosstalk of $\mathrm{Wnt} / \beta$-catenin/Bmp signaling within the apical ectodermal ridge (AER) regulates interdigit formation," Biochemical and Biophysical Research Communications, vol. 391, no. 4, pp. 1653-1657, 2010.

[11] R. Hernández-Martínez and L. Covarrubias, "Interdigital cell death function and regulation: new insights on an old programmed cell death model," Development, Growth \& Differentiation, vol. 53, no. 2, pp. 245-258, 2011.

[12] L. B. Corson, Y. Yamanaka, K.-M. V. Lai, and J. Rossant, "Spatial and temporal patterns of ERK signaling during mouse embryogenesis," Development, vol. 130, no. 19, pp. 4527-4537, 2003.

[13] C. J. Sheeba, I. Palmeirim, and R. P. Andrade, "Retinoic acid signaling regulates embryonic clock hairy2 gene expression in the developing chick limb," Biochemical and Biophysical Research Communications, vol. 423, no. 4, pp. 889-894, 2012.

[14] D. Ren, H.-C. Tu, H. Kim et al., "BID, BIM, and PUMA are essential for activation of the BAX- and BAK-dependent cell death program," Science, vol. 330, no. 6009, pp. 1390-1393, 2010.

[15] R. Hernandez-Martinez, S. Castro-Obregon, and L. Covarrubias, "Progressive interdigital cell death: regulation by the antagonistic interaction between fibroblast growth factor 8 and retinoic acid," Development, vol. 136, no. 21, pp. 3669-3678, 2009.

[16] D. R. McCulloch, C. M. Nelson, L. J. Dixon et al., "ADAMTS metalloproteases generate active versican fragments that regulate interdigital web regression," Developmental Cell, vol. 17, no. 5, pp. 687-698, 2009.
[17] X. Zhao, T. Brade, T. J. Cunningham, and G. Duester, "Retinoic acid controls expression of tissue remodeling genes Hmgn1 and Fgf18 at the digit-interdigit junction," Developmental Dynamics, vol. 239, no. 2, pp. 665-671, 2010.

[18] V. Dupé, N. B. Ghyselinck, V. Thomazy et al., "Essential roles of retinoic acid signaling in interdigital apoptosis and control of BMP-7 expression in mouse autopods," Developmental Biology, vol. 208, no. 1, pp. 30-43, 1999.

[19] Y. Li, B. Pawlik, N. Elcioglu et al., "LRP4 mutations alter wnt/ $\beta$-catenin signaling and cause limb and kidney malformations in cenani-lenz syndrome," The American Journal of Human Genetics, vol. 86, no. 5, pp. 696-706, 2010.

[20] B. I. Dimitrov, T. Voet, L. De Smet et al., "Genomic rearrangements of the GREM1-FMN1 locus cause oligosyndactyly, radio-ulnar synostosis, hearing loss, renal defects syndrome and Cenani-Lenz-like non-syndromic oligosyndactyly," Journal of Medical Genetics, vol. 47, no. 8, pp. 569-574, 2010.

[21] J. M. Verheyden and X. Sun, "An Fgf/Gremlin inhibitory feedback loop triggers termination of limb bud outgrowth," Nature, vol. 454, no. 7204, pp. 638-641, 2008.

[22] M. M. Al-Qattan, Y. Yang, and S. H. Kozin, "Embryology of the upper limb," The Journal of Hand Surgery, vol. 34, no. 7, pp. 1340-1350, 2009.

[23] S. D. Weatherbee, R. R. Behringer, J. J. Rasweiler IV, and L. A. Niswander, "Interdigital webbing retention in bat wings illustrates genetic changes underlying amniote limb diversification," Proceedings of the National Academy of Sciences, vol. 103, no. 41, pp. 15103-15107, 2006.

[24] S. Lohan, M. Spielmann, S. C. Doelken et al., "Microduplications encompassing the sonic hedgehog limb enhancer ZRS are associated with Haas-type polysyndactyly and Laurin-Sandrow syndrome," Clinical Genetics, vol. 86, no. 4, pp. 318-325, 2014.

[25] J. W. P. Potuijt, M. Baas, R. Sukenik-Halevy et al., "A point mutation in the pre-ZRS disrupts sonic hedgehog expression in the limb bud and results in triphalangeal thumb-polysyndactyly syndrome," Genetics in Medicine, vol. 20, no. 11, pp. 1405-1413, 2018.

[26] J. T. Eggenschwiler, E. Espinoza, and K. V. Anderson, "Rab23 is an essential negative regulator of the mouse sonic hedgehog signalling pathway," Nature, vol. 412, no. 6843, pp. 194-198, 2001.

[27] D. Krawchuk, S. J. Weiner, Y.-T. Chen et al., "Twist1 activity thresholds define multiple functions in limb development," Developmental Biology, vol. 347, no. 1, pp. 133-146, 2010.

[28] R. J. Lipinski, J. J. Gipp, J. Zhang, J. D. Doles, and W. Bushman, "Unique and complimentary activities of the Gli transcription factors in Hedgehog signaling," Experimental Cell Research, vol. 312, no. 11, pp. 1925-1938, 2006.

[29] R. Dobrowolski, G. Hertig, H. Lechner et al., "Loss of connexin43-mediated gap junctional coupling in the mesenchyme of limb buds leads to altered expression of morphogens in mice," Human Molecular Genetics, vol. 18, no. 15, pp. 2899-2911, 2009.

[30] M. M. Al-Qattan and M. A. Al-Husain, "Classification of hand anomalies in Apert's syndrome," Journal of Hand Surgery, vol. 21, no. 2, pp. 266-268, 1996.

[31] O. A. Ibrahimi, E. S. Chiu, J. G. McCarthy, and M. Mohammadi, "Understanding the molecular basis of Apert syndrome," Plastic and Reconstructive Surgery, vol. 115, no. 1, pp. 264-270, 2005.

[32] V. M. Fresco, C. B. Kern, M. Mohammadi, and W. O. Twal, "Fibulin-1 binds to fibroblast growth factor 8 with high 
affinity," Journal of Biological Chemistry, vol. 291, no. 36, pp. 18730-18739, 2016.

[33] M. M. Al-Qattan, "Type II familial synpolydactyly: report on two families with an emphasis on variations of expression," European Journal of Human Genetics, vol. 19, no. 1, pp. 112-114, 2011.

[34] P. Kuss, P. Villavicencio-Lorini, F. Witte et al., "Mutant Hoxd13 induces extra digits in a mouse model of synpolydactyly directly and by decreasing retinoic acid synthesis," Journal of Clinical Investigation, vol. 119, no. 1, pp. 146-156, 2009.

[35] N. Brison, P. Debeer, and P. Tylzanowski, "Joining the fingers: AHOXD13story,” Developmental Dynamics, vol. 243, no. 1, pp. 37-48, 2014.

[36] Y. Chen, V. Knezevic, V. Ervin, R. Hutson, Y. Ward, and S. Mackem, "Direct interaction with Hoxd proteins reverses Gli3-repressor function to promote digit formation downstream of Shh," Development, vol. 131, no. 10, pp. 2339-2347, 2004.

[37] D. Simon-Chazottes, S. Tutois, M. Kuehn et al., "Mutations in the gene encoding the low-density lipoprotein receptor LRP4 cause abnormal limb development in the mouse," Genomics, vol. 87, no. 5, pp. 673-677, 2006.

[38] A. Zuniga, O. Michos, F. Spitz et al., "Mouse limb deformity mutations disrupt a global control region within the large regulatory landscape required for Gremlin expression," Genes \& Development, vol. 18, no. 13, pp. 1553-1564, 2004.

[39] J. Capdevila, T. Tsukui, C. R. Esteban, V. Zappavigna, and J. C. I. Belmonte, "Control of vertebrate limb outgrowth by the proximal factor Meis2 and distal antagonism of BMPs by Gremlin," Molecular Cell, vol. 4, no. 5, pp. 839-849, 1999.

[40] U. Guha, W. A. Gomes, T. Kobayashi, R. G. Pestell, and J. A. Kessler, "In vivo evidence that BMP signaling is necessary for apoptosis in the mouse limb," Developmental Biology, vol. 249, no. 1, pp. 108-120, 2002.

[41] S. Pajni-Underwood, C. P. Wilson, C. Elder, Y. Mishina, and M. Lewandoski, "BMP signals control limb bud interdigital programmed cell death by regulating FGF signaling," Development, vol. 134, no. 12, pp. 2359-2368, 2007.

[42] A. Bandyopadhyay, K. Tsuji, K. Cox, B. D. Harfe, V. Rosen, and C. J. Tabin, "Genetic analysis of the roles of BMP2, BMP4, and BMP7 in limb patterning and skeletogenesis," PLoS Genetics, vol. 2, no. 12, p. e216, 2006.

[43] Y. L. Wong, R. R. Behringer, and K. M. Kwan, "Smad1/ Smad5 signaling in limb ectoderm functions redundantly and is required for interdigital programmed cell death," Developmental Biology, vol. 363, no. 1, pp. 247-257, 2012.

[44] K. Mouri, T. Sagai, A. Maeno, T. Amano, A. Toyoda, and T. Shiroishi, "Enhancer adoption caused by genomic insertion elicits interdigital Shh expression and syndactyly in mouse," Proceedings of the National Academy of Sciences, vol. 115, no. 5, pp. 1021-1026, 2018.

[45] Y. Pan, Z. Liu, J. Shen, and R. Kopan, "Notch1 and 2 cooperate in limb ectoderm to receive an early Jagged 2 signal regulating interdigital apoptosis," Developmental Biology, vol. 286, no. 2, pp. 472-482, 2005.

[46] R. Jiang, Y. Lan, H. D. Chapman et al., "Defects in limb, craniofacial, and thymic development in Jagged2 mutant mice," Genes \& Development, vol. 12, no. 7, pp. 1046-1057, 1998.

[47] Y. Lallemand, M. A. Nicola, C. Ramos, A. Bach, C. S. Cloment, and B. Robert, "Analysis of Msx1; Msx2 double mutants reveals multiple roles for Msx genes in limb development," Development, vol. 132, no. 13, pp. 3003-3014, 2005.
[48] D. P. Cavalcanti, V. Matejas, D. Luquetti, M. F. Mello, and M. Zenker, "Fraser and Ablepharon macrostomia phenotypes: concurrence in one family and association with mutated FRAS1," American Journal of Medical Genetics Part A, vol. 143A, no. 3, pp. 241-247, 2007.

[49] E. A. Hines, J. M. Verheyden, A. J. Lashua et al., "Syndactyly in a novel Fras1 rdf mutant results from interruption of signals for interdigital apoptosis," Developmental Dynamics, vol. 245, no. 4, pp. 497-507, 2016.

[50] J. Dai, B. Estrada, S. Jacobs et al., "Dissection of Nidogen function in Drosophila reveals tissue-specific mechanisms of basement membrane assembly," PLoS Genetics, vol. 14, no. 9, Article ID e1007483, 2018.

[51] K. Böse, R. Nischt, A. Page, B. L. Bader, M. Paulsson, and N. Smyth, "Loss of nidogen-1 and -2 results in syndactyly and changes in limb development," Journal of Biological Chemistry, vol. 281, no. 51, pp. 39620-39629, 2006.

[52] P. J. Bosch, L. C. Fuller, and J. A. Weiner, "An essential role for the nuclear protein Akirin2 in mouse limb interdigital tissue regression," Scientific Reports, vol. 8, no. 1, p. 12240, 2018.

[53] K. C. Oberg, "Classification of congenital upper limb anomalies: towards improved communication, diagnosis, and discovery," Journal of Hand Surgery (European Volume), vol. 44, no. 1, pp. 4-14, 2019.

[54] M. M. Al-Qattan, "Classification of dorsal and ventral dimelia in humans," Journal of Hand Surgery (European Volume), vol. 38, no. 9, pp. 928-933, 2013.

[55] M. M. Al-Qattan, "Classification of hand anomalies in Poland's syndrome," British Journal of Plastic Surgery, vol. 54, no. 2, pp. 132-136, 2001.

[56] M. M. Al-Qattan and A. Al Thunayan, "The middle phalanx in Poland syndrome," Annals of Plastic Surgery, vol. 54, no. 2, pp. 160-164, 2005.

[57] M. M. Al-Qattan and S. H. Kozin, "Update on embryology of the upper limb," The Journal of Hand Surgery, vol. 38, no. 9, pp. 1835-1844, 2013.

[58] S. S. Chaudhry, J. Gazzard, C. Baldock et al., "Mutation of the gene encoding fibrillin-2 results in syndactyly in mice," Human Molecular Genetics, vol. 10, no. 8, pp. 835-843, 2001.

[59] D. P. Reinhardt, T. Sasaki, B. J. Dzamba et al., "Fibrillin-1 and fibulin-2 interact and are colocalized in some tissues," Journal of Biological Chemistry, vol. 271, no. 32, pp. 19489-19496, 1996.

[60] O. Schatz, E. Langer, and N. Ben-Arie, "Gene dosage of the transcription factor Fingerin (bHLHA9) affects digit development and links syndactyly to ectrodactyly," Human Molecular Genetics, vol. 23, no. 20, pp. 5394-5401, 2014.

[61] E. Klopocki, S. Lohan, S. C. Doelken et al., "Duplications of BHLHA9 are associated with ectrodactyly and tibia hemimelia inherited in non-Mendelian fashion," Journal of Medical Genetics, vol. 49, no. 2, pp. 119-125, 2012.

[62] J. R. Collier, N. A. M. Monk, P. K. Maini, and J. H. Lewis, "Pattern formation by lateral inhibition with feedback: a mathematical model of delta-notch intercellular signalling," Journal of Theoretical Biology, vol. 183, no. 4, pp. 429-446, 1996.

[63] A. Murgai, S. Altmeyer, S. Wiegand, P. Tylzanowski, and S. Stricker, "Cooperation of BMP and IHH signaling in interdigital cell fate determination," PLoS One, vol. 13, no. 5, Article ID e0197535, 2018.

[64] E. Haro, I. Delgado, M. Junco et al., "Sp6 and Sp8 transcription factors control AER formation and dorsal-ventral patterning in limb development," PLoS Genetics, vol. 10, no. 8, Article ID e1004468, 2014. 
[65] M. Lewandoski, X. Sun, and G. R. Martin, "FGF8 signalling from the AER is essential for normal limb development," Nature Genetics, vol. 26, no. 4, pp. 460-463, 2000.

[66] P. Lu, G. Minowada, and G. R. Martin, "Increasing Fgf4 expression in the mouse limb bud causes polysyndactyly and rescues the skeletal defects that result from loss of Fgf8 function," Development, vol. 133, no. 1, pp. 33-42, 2006.

[67] M. A. Al Ghamdi, M. M. Al-Qattan, A. Hadadi et al., "A classification system for split-hand/foot malformation (SHFM): a proposal based on 3 pedigrees with WNT10B mutations," European Journal of Medical Genetics, Article ID 103738, 2019.

[68] M. M. Al-Qattan, "Central and ulnar cleft hands: a review of concurrent deformities in a series of 47 patients and their pathogenesis," Journal of Hand Surgery (European Volume), vol. 39, no. 5, pp. 510-519, 2014.

[69] T. Laurell, D. Nilsson, W. Hofmeister et al., "Identification of three novel FGF16 mutations in X-linked recessive fusion of the fourth and fifth metacarpals and possible correlation with heart disease," Molecular Genetics \& Genomic Medicine, vol. 2, no. 5, pp. 402-411, 2014.

[70] R. Nomura, E. Kamei, Y. Hotta, M. Konishi, A. Miyake, and N. Itoh, "Fgf16 is essential for pectoral fin bud formation in zebrafish," Biochemical and Biophysical Research Communications, vol. 347, no. 1, pp. 340-346, 2006.

[71] Y. Wang, R. Xiao, F. Yang et al., "Abnormalities in cartilage and bone development in the Apert syndrome FGFR2+/ S252W mouse," Development, vol. 132, no. 15, pp. 3537-3548, 2005.

[72] M. M. Al-Qattan, “A unique case of Cenani-Lenz type syndactyly without flexor or intrinsic function," Journal of Hand Surgery, vol. 31, no. 4, pp. 460-462, 2006.

[73] T. N. Khan, J. Klar, Z. Ali, F. Khan, S. M. Baig, and N. Dahl, "Cenani-Lenz syndrome restricted to limb and kidney anomalies associated with a novel LRP4 missense mutation," European Journal of Medical Genetics, vol. 56, no. 7, pp. 371-374, 2013.

[74] A. Kariminejad, B. Stollfuß, Y. Li et al., "Severe Cenani-Lenz syndrome caused by loss of LRP4 function," American Journal of Medical Genetics Part A, vol. 161, no. 6, pp. 1475-1479, 2013.

[75] A. S. Lindy, C. P. Bupp, S. J. McGee et al., "Truncating mutations in LRP4 lead to a prenatal lethal form of Cenani-Lenz syndrome," American Journal of Medical Genetics Part A, vol. 164, no. 9, pp. 2391-2397, 2014.

[76] M. Afzal, Q. Zaman, U. Kornak, S. Mundlos, S. Malik, and R. Flöttmann, "Novel splice mutation in LRP4 causes severe type of Cenani-Lenz syndactyly syndrome with oro-facial and skeletal symptoms," European Journal of Medical Genetics, vol. 60, no. 8, pp. 421-425, 2017.

[77] D. M. Ibrahim, N. Tayebi, A. Knaus et al., "A homozygous HOXD13 missense mutation causes a severe form of synpolydactyly with metacarpal to carpal transformation," American Journal of Medical Genetics Part A, vol. 170, no. 3, pp. 615-621, 2016.

[78] M. M. Al-Qattan, "Preaxial polydactyly of the upper limb viewed as a spectrum of severity of embryonic events," Annals of Plastic Surgery, vol. 71, no. 1, pp. 118-124, 2013. 


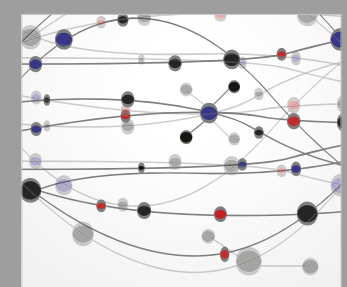

The Scientific World Journal
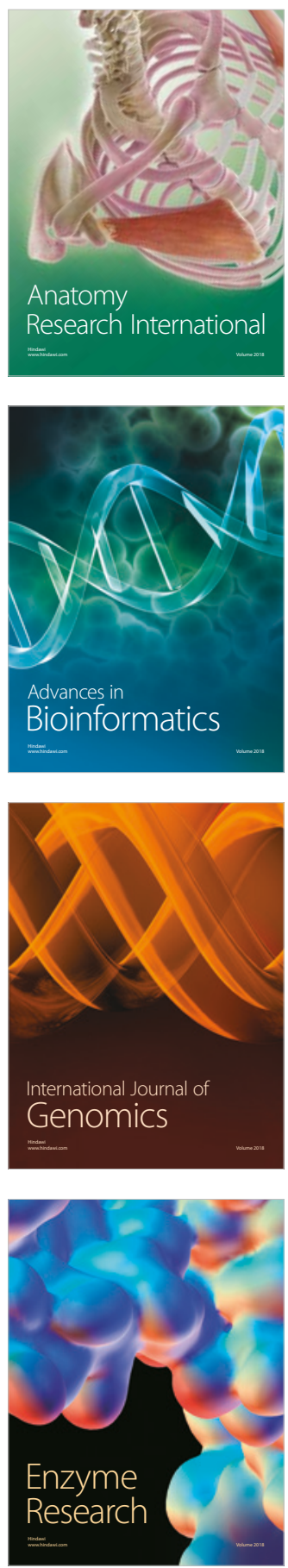
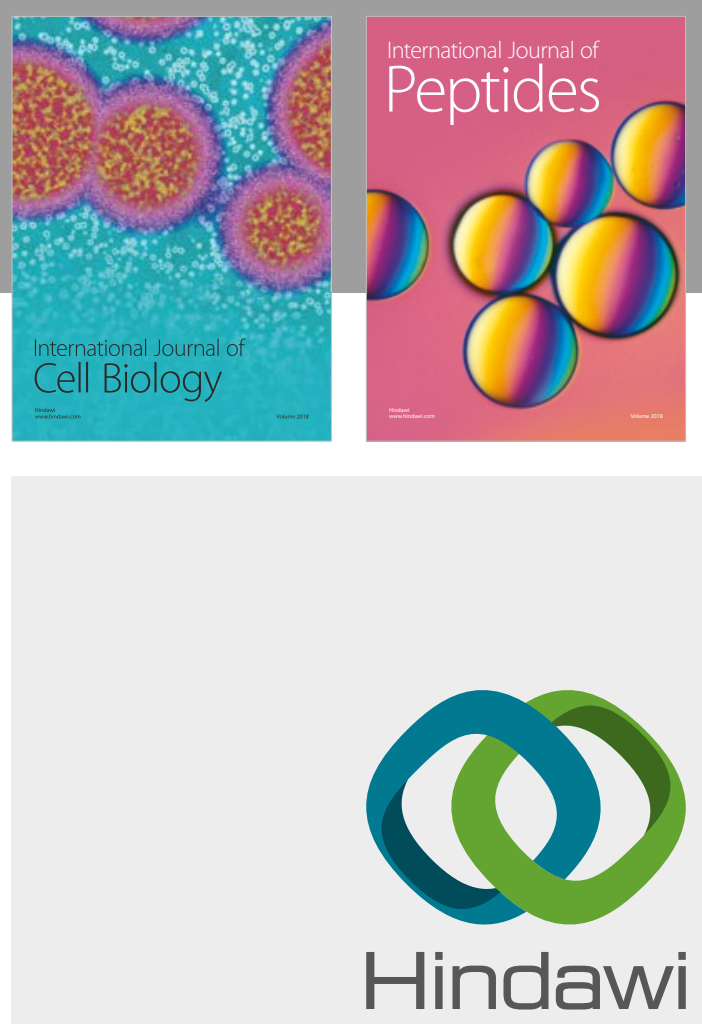

Submit your manuscripts at

www.hindawi.com
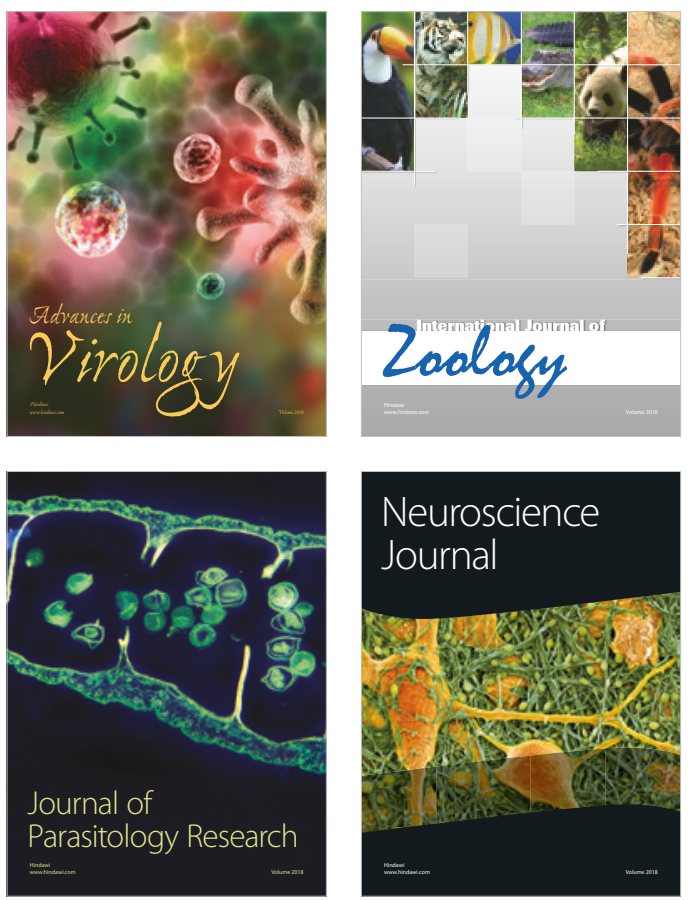
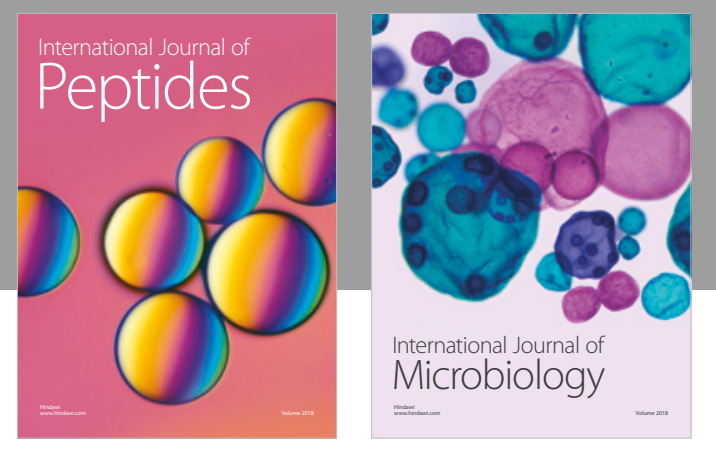

nternational Journal of Microbiology
Journal of
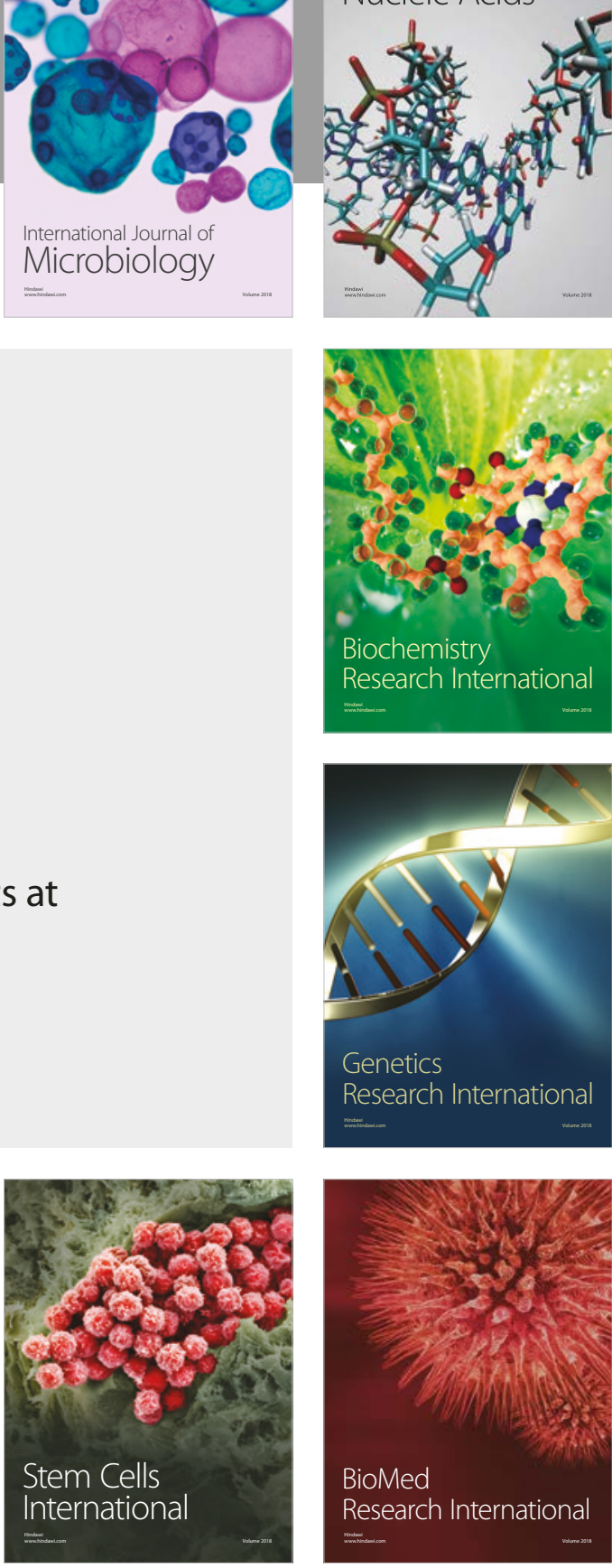
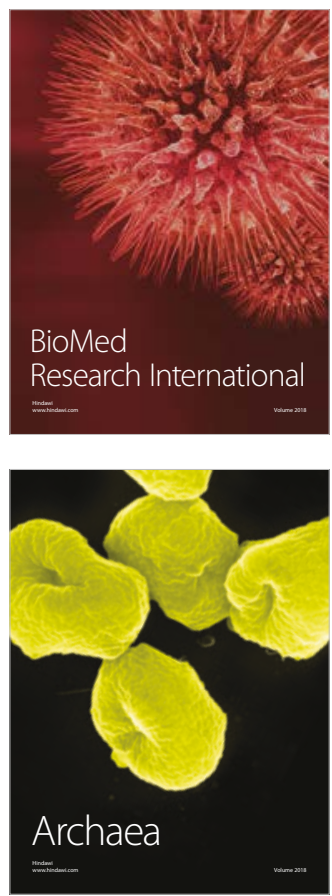